\title{
BIBLIOGRAFÍA SOBRE JUEGOS POPULARES Y EDUCACIÓN EN LA HISTORIA
}

\section{Bibliography on popular games and education in history}

Juan Francisco Cerezo Manrique

Universidad de Salamanca

Correo-e: jfcm@usal.es

Miguel Ángel Cerezo Manrique

Universidad de Valladolid

Correo-e: migelangelcerezo@hotmail.com

Recepción: 5 de enero de 2019. Envío a informantes: I2 de enero de 2019

Aceptación definitiva: 27 de febrero de 2019

Abril, E.: Dos siglos de pelota vasca, Madrid, I97I.

Adams, S.: El fantástico libro de los juegos, Barcelona, Círculo de lectores, 1993.

Aficionado: Lícito recreo casero, ó coleccion de cincuenta juegos conocidos comunmente con el nombre de juegos de prendas, Madrid, Imp. Ramón Ruiz, I792.

Agramonte, E. A.: «Juegos y deportes populares y tradicionales», Pedagogía Magna, in (20II), pp. 98-IO8.

Agudo Brigidano, D.; Minués Pérez, R.; Rojas García, C.; Ruiz Fernández, M.; SalvaDor Minués, R. y Tomás Larrén, J.: Juegos de todas las culturas, Barcelona, Inde, 2002.

Aguirre, R.: Deporte rural vasco, San Sebastián, Txertoa, 1983.

Ajuntament de Barcelona: Els jardins dels infants, Barcelona, Comissió de Cultura de l'Ajuntament de Barcelona, I920.

Ajuntament de Barcelona: Escoles a l'aire lliure del Parc de Montjuic, Barcelona, Ajuntament de Barcelona, I92I.

Alcocer, F. de: Tratado del juego, Salamanca, I559.

Allué, J. M.: El gran libro de los juegos, Barcelona, Editorial Parramón, 1998.

Allué, J. M.: Juegos para todo el año. roo juegos para el otoño, Barcelona, Editorial Parramón, I999.

Almeida, A.: «Escultismo y Educación Física en Canarias», Vegueta. Anuario de la Facultad de Geografía e Historia, Servicio de Publicaciones de la Universidad de Las Palmas de Gran Canaria, 8 (2004), pp. 5I-69. 
Almeida, A.: Las colonias escolares en Gran Canaria (1922-1936). Textos e imágenes para su estudio, Mercurio, Madrid, 2018.

Alonso, M. R.: Un rincón tinerfeño. La Punta del Hidalgo, La Laguna, Real Sociedad Económica de Amigos del País de Tenerife, 1944.

Álvarez, F.: «Investigar el juego», Cuadernos de Pedagogía, 99 (1983).

Álvarez, J. M.: «El juguete como instrumento educativo», Vida Escolar, 202 (1982).

Amades, J.: "Cultura i jocs infantils», en Folklore de Catalunya, Barcelona, Biblioteca Selecta, 1980.

Amar, J. y Jauffret, L.: La gimnástica ó escuela de juventud, Madrid, Imp. de Álvarez, I807.

Amat, M. y Batalla, A.: "Deporte y educación en valores», Aula de Innovación Educativa, 9I (2000), pp. IO-I3.

Amonachvili, Ch.: «El juego en la actividad de aprendizaje de los escolares», Perspectivas UNESCO, vol. XVI, I (1986).

Amorós, J.: «Juegos y ejercicios de gimnasia al aire libre que la autora hace practicar con preferencia a los párvulos de su escuela y fundamento pedagógico de esta preferencia», Archivo de la Diputación Provincial de Valencia, E.9.3.15, caja I, 1908, p. I7.

Andrés Martín, F.: Juegos y deportes autóctonos, Salamanca, Ediciones de la Diputación de Salamanca, 1987.

Arranz, J. D.: Juegos al aire libre. Educación infantil y primaria, Madrid, Editorial Escuela Española, I995.

Aymerich, M.: «El juego», La escuela en acción (suplemento de El Magisterio Español), Io.089 (1972).

BALdó, M.: «El folk-lore en la escuela», Revista de Pedagogía, 43 (1925).

Ballesteros, A.: «El juego y el trabajo en la educación», Revista de Pedagogía, I33 (1933).

Bandet, J. y Sarazanas, R.: El niño y sus juguetes, Madrid, Narcea, 1978.

BAntulá, J.: Juegos motrices cooperativos, Barcelona, Paidotribo, I998.

BAntulà, J.: «El Joc popular i tradicional als segles XVI i XVII: revisió des d'una perspectiva taxonómica», Educació i història: Revista d'història de l'educació, 8 (2005), pp. I34-I46.

BANTULÀ, J.: «Los estudios socioculturales sobre el juego tradicional: una revisión taxonómica», Revista de Dialectología y Tradiciones Populares, 6I (2) (2006), pp. 19-42.

BAntulà, J.: «Per una pedagogia del joc: la seva aposta educativa des del segle XIX», Revista catalana de pedagogia, 6 (2007-2008), pp. 36I-380.

BANTULÀ, J.: «Una mirada a l'home que juga», Aloma: revista de psicologia, ciències de l'educació $i$ de l'esport, 25 (2009), pp. 15-27.

BANTULÀ, J.: «En torno al "enseñar deleitando" de Horacio", en La educación revisitada: ensayos de hermenéutica pedagógica, Moreu, Ángel y Prats Gil, Enric, (coords.): 20IO, pp. 59-69.

BANTUlà, J. y Mora, J. M.: Juegos multiculturales: 225 juegos tradicionales para un mundo global, Barcelona, Paidotribo, 2002.

Bantulà, J. y Vilanou, C.: «Joc, humanisme i pedagogia: la virtut de l>eutrapèlia», Aloma: revista de psicologia, ciències de l'educació $i$ de l'esport, 25 (2009), pp. 53-89.

Barandiarán, J. M. y Manterola, A (eds.): Atlas etnográfico de Vasconia. Juegos infantiles en Vasconia, Bilbao, Erniker Euskleria, 1993.

Barceló, J. y Forteza, Ll.: Cançons i jocs populars de les Illes Balears, Palma de Mallorca, Centre d'Estudis d'Esplai, 1993 .

BARnés, D.: «Escuelas al aire libre», Boletín de la Institución Libre de Enseñanza, 605 (1910).

BARnés, Domingo: "La educación física y el juego», Boletín de la Institución Libre de Enseñanza, 784 (1925), pp. 199-203.

Bartolomé, E.: «El concurso de El Liberal», La Escuela Moderna, II2 (1900).

BARreau, J.: «Juegos tradicionales, ética deportiva e ideologías del mercado», Revista de Educación Física: Renovar la Teoría y Práctica, I42 (2016), pp. 37-42. 
Bastinos, J.: Juegos infantiles. Recreos infantiles para la infancia y la juventud, Barcelona, Librería de Antonio J. Bastinos, I895.

Bastinos, J.: Juegos Infantiles, Barcelona, Lib. de Antonio J. Bastinos, I896.

BArreto, M.: «Construyendo identidades: juegos y deportes tradicionales canarios», en AmADoR, F. y otros: Luchas. Deportes de combate y juegos tradicionales, Madrid, Gymnos, 1996.

Bassedas, E.; Rosell, M. y Solé, I.: «Juego y trabajo en el parvulario», Cuadernos de Pedagogía, 8I-82 (198I).

BAtAller, J.: Els jocs dels xiquets al País Valencià, Valencia, ICE Universitat de València, 1979.

Bautista, J. E.: El juego y deporte popular, tradicional y autóctono en la escuela. Los bolos buertanos y los bolos cartageneros, Wanceulen SL, 2008.

BengoA, J. L.: Juegos vascos, Bilbao, Caja de Ahorros Vizcaína, i98I.

Blanchard, K. y Cheska, A.: Antropología del deporte, Barcelona, Bellaterra, 1986.

Blanco, R.: Tratado elemental de Pedagogía, Madrid, Tipografía de la Revista de Archivos, Bibliotecas y Museos, I9I4.

Blanco García, T.: Para jugar como jugábamos: Colección de juegos y entretenimientos de la tradición, Salamanca, Diputación Provincial de Salamanca, Centro de Cultura Tradicional, 1993.

Blanco SuÁrez, P.: «Los ejercicios y juegos corporales en España», Boletín de la Institución Libre de Enseñanza, 413 (1894), pp. 227-234.

Bordgna, F.: «Juego y conocimiento», Cuadernos de Pedagogía, 99 (1983).

BorJa, M. de: El juego como actividad educativa. Instruir deleitando, Barcelona, Publicaciones de la Universidad de Barcelona, 1984.

Borja, M. de: Las ludotecas. Instituciones de juegos, Barcelona, Octaedro, 2000.

Brasó, J.: El joc, un element clau en la història de la renovació pedagógica, tesis doctoral, Universitat de Barcelona, 2017.

Brasó, J.: «Pere Vergés: escuela y gamificación a comienzos del siglo xx», Apunts: Educación física y deportes, 133 (2018), pp. 20-37.

Brasó, J. y Collell, X.: «El joc popular de la xarranca. Estudi a través dels llibres i del folklore catalá. Possibles aplicacions en l'ambit escolar», REIRE: revista d'innovació i recerca en educació, vol. 9, 2 (2016), pp. 82-105.

Brasó, J. y Torrebadella, X.: «El joc del rescat a Catalunya. Un projecte educatiu a l'Escola del Mar de Pere Vergés», Temps d'educació, 47 (20I4), pp. I9I-2I2.

Brasó, J. y Torrebadella, X.: «Análisi i classificació dels jocs de la infancia de Joan Amades en funció de la seva lógica interna i del gènere dels practicants (I674-I947)», REIRE: revista d'innovació i recerca en educación, vol. 8, 2 (2015), pp. I8-4I.

Brasó, J. y Torrebadella, X.: «El marro, un juego tradicional y popular en la educación física española (1807-1936)», Revista Complutense de Educación, vol. 26, 3 (2015), pp. 697-719.

Brasó, J. y Torrebadella, X.: «El juego motor del marro: una indagación acerca de sus raíces pedagógicas», Revista de Dialectología y Tradiciones Populares, tomo 72, Cuaderno I (2017), pp. 245-264.

Brasó, J.: «Thomas Arnold, Pere Vergés i els jocs organitzats. Els scacs, un projecte educatiu a l'Escola del Mar», Temps d'educació, 49 (2015), pp. 135-I63.

Bravo Villasante, C.: Una dola, tela catola. El libro del folklore infantil, Madrid, Miñón, 1977.

Bravo Villasante, C.: China, China, Capuchina, en esta mano está la china, Madrid, Miñón, I98I.

Bravo Villasante, C.: Colorín, colorete, Madrid, Didascalia, 1983.

Buitendijk, F.: El juego y su significado, Madrid, Rev. de Occidente, 1936.

Burne, C.: «La Ciencia del Folk-Lore», Boletín de la Institución Libre de Enseñanza, 209 (I885), pp. 310-313.

Buylla, A.: «El libro de M. Daryl sobre la educación física», Boletín de la Institución Libre de Enseñanza, 296 (1889), pp. I62-163.

Buylla, A.: «Nota sobre los juegos corporales», Boletín de la Institución Libre de Enseñanza, 3I7 (I889), pp. II8-I2O. 
C. R.: Juegos de jardin o campo, Madrid, R y Fonseca, I847.

Caballe, J. y Ruscadella, T.: Recull de jocs popular gironins, Girona, Servei Municipal de Publicacions, 1982.

Caillois, R.: Teoría de los juegos, Barcelona, Seix Barral, 1958.

CAmpomanes, V.: Un estudio de los juegos populares y los deportes tradicionales en la provincia de Valencia, tesis inédita, Madrid, INEF, 1983.

Carbonell Sánchez, M.: «Niños y juegos», La Escuela Moderna, viI, 74 (1897).

Carbonell, M.: «Pedagogía maternal. IV. Juegos y juguetes», La Escuela Moderna, I48 (1903).

Cardedera, M.: Diccionario de educación y métodos de enseñanza. Tomo iII, Madrid, Imprenta de A. Vicente, 1856.

Caro, R.: Días geniales o lúdricos, Madrid, Espasa-Calpe, 1978.

Carranza, M. y Mora, J. M.: Educación física y valores: educando en un mundo complejo, Barcelona, Graó, 2003.

Casal, J. y Fuentes, X.: Costumbres populares: Apuntes acerca de juegos, diversiones, pasatiempos, entretenimientos y juguetes de mujeres y hombres, juveniles y de muchacho, Santiago de Compostela, Consello da Cultura Galega, 2008.

Castellanos, B. S.: «De los juegos infantiles de los niños y su origen», Museo de los Niños, Madrid, t. II, I848.

Castiella, F.: «Los juegos infantiles», Revista de Pedagogía, 92 (1929).

Castro, N.: «Educación física de las niñas», La Escuela Moderna, 49 (I895).

Castro, U.: «Los juegos y los deportes tradicionales de Canarias en la Educación Física Escolar», en Hernández Moreno, J.; Rodríguez Ribas, J. P.; Ramírez, V.; Quiroga, M. y Castro, U. (coords.): Educación Física Escolar y Deporte de Alto rendimiento, Las Palmas de Gran Canaria, ACCAFIDE, I998, pp. II3-I32.

Castro, U.: Estudio etnográfico y de la lógica de las situaciones motrices de un juego tradicional desaparecido: la pina, tesis doctoral, Las Palmas de Gran Canaria, Universidad de Las Palmas de Gran Canaria, 20oI.

Castro, U.: «Del juego a la lúdica tradicional: el caso de Canarias», Recuerdos y Sensaciones. Álbum Artístico del Deporte, Las Palmas de Gran Canaria, Universidad de Las Palmas de Gran Canaria, 2004, pp. 63-89.

Castro, U.: «Los juegos y deportes tradicionales de Canarias. Una realidad social y cultural del siglo xxI», en Dueñas, E. X. (ed.): Juegos, Deportes e Investigación folclórica, Donostia, Eusko Ikaskutza, 2009.

Castro Guisasola, F.: Canciones y Juegos de los niños de Almería, Almería, Caja de Ahorros de Almería, I973.

Causí, T.: Bosquejo de una teoría biológica del juego infantil, Madrid, Calpe, 1924.

Chaves, R.: «La educación física en la escuela. El juego o la actividad lúdica predeportiva», La Escuela en Acción, 8.197 y 8.198 (1954).

Chateau, J.: Psicología de los juegos, Buenos Aires, Kapelusz, I968.

Claret, M.: Juegos de ayer y de hoy, Barcelona, Editorial Juventud, 1983,

Colomer, E.: «Indicación de los juegos infantiles más comunes y consideraciones sobre su valor educativo», $A D P V$, E.9.3.15, caja I, p. I6.

Columbié, R.: «Los juegos», Boletín de la Escuela Moderna, II, I (1902).

«Conclusiones del II Congreso Internacional sobre el juego y el juguete en el ámbito de la escuela y la educación especial», Bordón, 222 (1978).

Confederación de Juegos y Deportes Vascos: Juegos y deportes vascos. Reglamento, s. 1/s. a.

Corral, M. A.: Juegos infantiles cordobeses de tradición oral, Córdoba, Diputación Provincial, 1984.

Corredor, J.: La joguina a Catalunya, Barcelona, Edicions 62, I98I.

Corredor, J.: «Algunas reflexiones», Cuadernos de Pedagogía, 99 (1983). 
Corredor, J.: El juguete en España, Madrid, Espasa-Calpe, I999.

Cortizas, A.: «El juego (tradicional) en la escuela», Padres y Maestros, 293 (2005).

Cossío, M. B.: «Los Museos de partido o cantonales», Boletín de la Institución libre de Enseñanza, t. v, II6 (I88I).

Cossío, M. B.: «Contra la introducción de los ejercicios militares y batallones escolares en la escuela», Boletín de la Institución Libre de Enseñanza, 272 (I888), pp. I45-I47.

Cousinet, R.: «El placer y el juego», Revista de Pedagogía, vi, 68 (1927).

Cruz Feliu, J.; Boixadós Anglés, M.; Torregrosa Álvarez, M. y Mimbrero Palop, J.: « ¿Existe un deporte educativo? Papel de las competiciones deportivas en el proceso de socialización del niño», Revista de Psicología del Deporte, 9-Io (I996), pp. III-I32.

Cuadernos de Pedagogía: «II Congreso Internacional sobre el juego y el juguete», Cuadernos de Pedagogía, 37 (1978).

Cuesta, E.: El habla y juegos populares en Macotera, Salamanca, 2006.

Curiel, M.: «Juegos infantiles de Extremadura», Revista Dialectología y Tradiciones Populares, I (1944), pp. I62-187.

CurTis, H. S.: «El juego y la formación de los hábitos y del carácter», Boletín Institución Libre de Enseñanza, 702 (1918).

Cuscoy, L. D.: Folklore infantil. Tradiciones populares, La Laguna, Inst. de Estudios Canarios, 1943.

De la Fuente, M.: Juegos populares en Cuenca, Cuenca, Ayuntamiento de Cuenca, 1985.

De los Reyes, I.: «Terminología del Folk-Lore», Boletín de la Institución Libre de Enseñanza, 205 (I885), pp. $25 \mathrm{I}-252$.

De SAn Martín, A.: «De los juegos corporales más convenientes en España», Boletín de la Institución Libre de Enseñanza, 303 (1889), pp. 273-275.

Decreto 2061/1969, de I6 de agosto, por el que se regula la clasificación del juguete educativo didáctico, $B O E$ del 29 de septiembre de 1969.

«Del Congreso de Higiene», Diario de Tenerife, 30 de mayo de i898, p. 2.

Del Pozo, C.: «Juguetes..., para que jueguen los niños», Escuela Española, 346 (1948).

Deportes autóctonos en Castilla y León, Valladolid, Junta de Castilla y León, 1985.

DíAz, J.: Cien temas infantiles, Valladolid, C. Castellano de Estudios Folklóricos, 198ז.

Díaz, J. y Martín, M.: Pasatiempos tradicionales, Valladolid, Castilla Ediciones, 1989.

Diego Cuscoy, L. Tradiciones Populares II: Folklore infantil, La Laguna, Instituto de Estudios Canarios, I943.

«Diversiones y cultura», Escuela Española, 378 (1948).

Dunyó, F.: «Jugar en la escuela», Cuadernos de Pedagogía, 57 (1979).

Durán González, J.: «La actividad física y el deporte: una oportunidad para transmitir valores», en A.A. v.v.: Valores en movimiento. La actividad física y el deporte como medio de educación en valores, Madrid, Ministerio de Educación y Ciencia. Consejo Superior de Deportes, 2006.

«El juego como manifestación social y cultural (juegos populares, autóctonos y tradicionales) [...] Valoración de los juegos populares, autóctonos y tradicionales como vínculo y parte del patrimonio cultural de la comunidad» Consejería de Educación, Cultura y Deportes, Decreto 46/I993, de 26 de marzo, por el que se establece el currículo de la Educación Primaria, Santa Cruz de Tenerife (BOC n. ${ }^{\circ} 44$ de viernes 9 de abril de 1993).

«El juego en la cultura física», El Tiempo, n. ${ }^{\circ} 3203,28$ de octubre de i9ı, p. I.

Eleizgui, J. de: Los juegos en la infancia (Guía médicopedagógica para padres y maestros, Barcelona, Sociedad general de publicaciones, s. a.

EsCARTí, A.: Responsabilidad personal y social a través de la educación física y el deporte, Barcelona, Graó, 2005.

EsCRIBANO, M. L.: Juegos infantiles granadinos de tradición oral, Granada, Universidad de Granada, I994. 
Etniker-Biscaia (ed.): Juegos y canciones infantiles. Colección temas vizcainos, Bilbao, Caja de Ahorros Vizcaína, 1979.

Eтxebeste, J.: «Jolasa y Jokoa: los dos conceptos vascos para el juego», en Actas del V Congreso de las ciencias del deporte, educación física y recreación, $200 \mathrm{I}$.

Felipe Maso, J. L.: «La Institución Libre de Enseñanza: sus principios pedagógicos innovadores y su presencia en el currículo de la educación física actual. El primer centro docente español que utilizó el deporte como elemento educativo", Citius, Altius, Fortius, 7 (2) (2015), pp. 57-82.

Fernández, Á.: Los juegos de las diferentes edades, en todos los pueblos del mundo, desde la antigüedad más remota hasta nuestros días. Primera Serie. Juegos y diversiones de la infancia y de la juventud, Madrid, Biblioteca Universal, I85I.

Fernández, F.: Los juegos de la primera edad, Madrid, Est. Tip. de Mellado, I862.

Fernández, J. E. R.; Couto, J. M. P. y Aguilar, J. P.: «La promoción de juegos y deportes populares y tradicionales: De los centros educativos al servicio municipal de deportes. El caso de Boiro», Revista Española de Educación Física y Deportes, 4I2 (2016), pp. 79-92.

Fernández Gamboa, A.: Los bolos en España, Gijón, Bankunión, 1978.

Ferraz, M.: «Las propuestas educativas libertarias de José Cabrera Díaz», Historia de la Educación, I7 (1998), pp. 33I-347.

Flores, A.: Ayer, hoy y mañana, Madrid, Montaner y Simón, I892.

Flores, C.: «El juego y los juguetes educativos para niños en edad preescolar», Didascalia, 8 (1970).

Folklore: Folklore a l'escola bressol, Dossier «Rosa Sensat», Barcelona, 1986.

FRAGUAS, J. E.: Tratado racional de gimnástica y de los ejercicios y juegos corporales practicables sin aparatos y con ellos en las casas particulares, gimnasios, jardines y en el campo para uso de las Universidades, Institutos, Escuelas Normales y Municipales, Academias, Colegios, Hospitales, Sociedades de recreo, etc., etc. Tomo III. Pedagogía general, Educación física y Juegos corporales, Madrid, Librería de la Viuda de Hernando y Cía, i896.

Fullat, O.: Educació i joc, Barcelona, Institut d'Estudis Catalans, 1996.

García, J.: «Juegos de niñas», Educación Pintoresca, 42 (I858), pp. I19-I22.

García, I.: «La importancia del juego en la infancia (I)», Escuela Española, 2.505 (1979).

García, M. A.: «La historia cercana a través del juego», Cuadernos de Pedagogía, in8 (1984).

García, M. E. y Ruiz, F.: Educación motriz a través del juego, Madrid, Gimnos, 20or.

García, P. de A.: Teoría y práctica de la educación y de la enseñanza: curso completo y enciclopédico de pedagogía, expuesto conforme a un método rigurosamente didáctico. Tomo v. De la educación física, Madrid, Librería de Hernando y Cía, I882.

García, P. de A.: «El renacimiento de la educación física», La Escuela Moderna, i (I89i), pp. 486-487.

García, P. de A.: Tratado de Pedagogía, Madrid-México, Saturnio Calleja-Herrero Hermanos, 1895 .

García Benítez, Á: El folklore infantil andaluz: antología de juegos populares, Sevilla, Editoriales Andaluzas Unidas, 1988.

García Beneitez, A.: Antología de juegos populares de Jaén, Jaén, Diputación Provincial de Jaén, 1990.

García Blanco: La lucha leonesa, León, Ed. F.F. Bernardino Sahagún, 1977.

García Rodríguez, C.: Juegos de nuestra tierra. Recopilación de juegos tradicionales de la provincia de Guadalajara, Sigüenza, Editorial Librería Rayuela, 1999.

García del Real, M.: «Lo que deben ser los juegos de los niños», La escuela Moderna, x, iI (I900).

García Serrano, R.: «Juegos y deportes de los españoles en los siglos xvi y xviI. Textos», en Cátedras universitarias de tema deportivo y cultural, Madrid, DNEF, 1973.

García Serrano, R.: Juegos y deportes tradicionales de España, Madrid, Inef de Madrid, I974. 
García Viciens, L.: Juegos tradicionales aragoneses II, Zaragoza, Librería General, 1978.

Garralda, D.: La educación física en la escuela. Gimnasia y juegos, Madrid, Espasa-Calpe, I94I. Generelo, E. y Planas, C.: «Cuéntame yayo: un ejemplo de material didáctico como apoyo a la formación del maestro acerca de los valores pedagógicos de los juegos tradicionales», en Amador, F. y otros: Luchas. Deportes de combate y juegos tradicionales, Madrid, Gymnos, I996.

Gil García, B.: Jugar y cantar, Madrid, Aguilar, i96I.

Giménez, A. M. y Fernández-Río, J.: «Los juegos tradicionales infantiles: Un marco privilegiado para el trabajo interdisciplinar y competencial», Tándem: Didáctica de la Educación Física, 33 (2010), pp. 67-76.

Giménez, A. M. y Río, J. F.: «Análisis y modificación de los juegos y deportes tradicionales para su adecuada aplicación en el ámbito educativo», Retos: Nuevas Tendencias en Educación Física, Deporte y Recreación, 19 (2011), pp. 54-58.

Giner DE Los Ríos, F.: «El ayuntamiento de Madrid y el juego de los niños», Boletín de la Institución Libre de Enseñanza, 38I (1887), pp. 338-339.

Giner DE los Ríos, F.: «Notas pedagógicas. Los problemas de la Educación física», Boletín de la Institución Libre de Enseñanza, 273 (1888), pp. I57-158.

Giradles, A. y Pelegrín, G.: Otro pueblos, otras culturas. Música y juegos del mundo, Madrid, Editorial MEC, 1996.

Giró, J.: Juegos infantiles de La Rioja, Zaragoza, Ibercaja, I990.

Gómez i Navarro, A.: «Juegos y deportes tradicionales. Narria», Estudios de Artes y Costumbres Populares, 65 (1994), pp. 59-64.

González GiL, M. D.: Cantar y jugar, Sevilla, Bibliografía infantil y juvenil de Andalucía, i984.

González Gil, M. D.: Palabras para jugar (Retahilas, trabalenguas, adivinanzas), Sevilla, Grazalema, 1993.

Gorris, J. M.: El juego y el juguete, Madrid, Queimada, 198I.

Gracia, L.: Juegos tradicionales aragoneses I, Zaragoza, 1978.

Grunfeld, F. V.: Juegos de todo el mundo, Madrid, Edilán, 1978.

Grupo Adarra: En busca del juego perdido, Bilbao, Adarra, 1984.

Grupo Corvera de Asturias: Juegos de ayer... para niños de hoy, La Morgal, Grupo Corverá de Asturias, 1997.

Guimerá, I.: «Los ejercicios corporales», Boletín de la Institución Libre de Enseñanza, 288 (I889), pp. 33-37.

Hernández, J.; Castro, U. y Navarro, V.: Los juegos y deportes tradicionales de Canarias, Las Palmas de Gran Canaria, Consejería de Educación, Cultura y Deportes, Universidad de Las Palmas de Gran Canaria, 2003.

Hernández, J. L.; Herrera, S. y López, J. M.: Antentú la de la falda azul: Hojas de folclore infantil de Ávila, Ávila, Fundación Cultural Santa Teresa, 2003.

Hernández Moreno, J. y Sánchez García, S.: Lucha canaria para niños y principiantes, Las Palmas de Gran Canaria, Hernández Moreno, I979.

Hernández de la Rosa, E.: «Colaboración social con el Maestro», Escuela Azul, Santa Cruz de Tenerife, n. ${ }^{\circ}$ 408, ro de agosto de 1950.

Hernández de Soto, S.: «Juegos infantiles de Extremadura», en Machado, Antonio (dir.): Biblioteca de las tradiciones populares españolas, t. II, Madrid, Librería de Fernando Fé, I884, pp. I09-I95.

Hernández de Soto, S.: "Juegos infantiles de Extremadura», en Machado, Antonio (dir.): Biblioteca de las tradiciones populares españolas, t. III, Madrid, Librería de Fernando Fé, I884, pp. 87-205.

Hernández de Soto, S.: Juegos infantiles de Extremadura, Introducción y edición de Salvador Rodríguez Becerra y Javier Marcos Arévalo, Mérida, Editora Regional de Extremadura, 1988 . 
Herrero, J.: Juegos infantiles asturianos: Recogidos de la tradición popular, Oviedo, Consejo Regional de Asturias, Consejería de Cultura y Deportes, I980.

Homs, E.: «La educación extra-escolar», Boletín de la Institución Libre de Enseñanza, 629 (I912). Huizinga, J.: Homo ludens, Madrid, Revista de Occidente, 1938.

IDÁÑEZ, A. F.: «Juegos y deportes tradicionales serranos: Los bolos y la bolera», Boletín del Instituto de Estudios Giennenses, 147 (1993), pp. 20I-233.

IRIgoYen, J.: El juego de la pelota a mano, Madrid, 1926.

JACQUIN, G.: La educación por el juego, Madrid, Atenas, 1958.

JARÉs, X.: El placer de jugar juntos. Juegos cooperativos, Madrid, Editorial ccs, 1992.

Jentzer, K.: Juegos educativos al aire libre y en la casa, Madrid, Francisco Beltrán, I92I.

Jiménez, J.: El juego de los bolos en Álava, Vitoria, Diputación Foral de Álava, I97o.

Jiménez De Madariaga, C.: «Sobre el folklore de Antonio Machado y Álvarez», en Actas del III Congreso de historia de la antropología y antropología aplicada. Pontevedra, I4-I6 de noviembre 1996, Pontevedra, Consejo Superior de Investigaciones Científicas, Csic, Instituto de Estudios Gallegos «Padre Sarmiento», 1997, pp. 197-208.

JiméNEZ VAQUeRizo, E.: «Juego y deporte tradicional en las clases de Educación Física (ESO) en la provincia de Segovia. Análisis de espacios y materiales», EFDeportes.com, Revista D $i$ gital, Buenos Aires, año I8, n. ${ }^{\circ}$ I83 (agosto de 20I3).

Jiménez Vaquerizo, E.: «El juego de bolos de Abades, patrimonio cultural y deportivo de Segovia (Deportes Autóctonos de Castilla y León)», EFDeportes.com, Revista Digital, Buenos Aires, año I8, n. ${ }^{\circ}$ I86 (noviembre de 2013).

Jiménez Vaquerizo, E.: «El origen del juego y deporte tradicional en Segovia (Castilla y León)», EFDeportes.com, Revista Digital, Buenos Aires, año I9, n. ${ }^{\circ}$ I95 (agosto de 2014).

J. N. H.: "Los juegos», La vida en la escuela (suplemento de Escuela Española), 820 (1956).

JorDÁn, J.: «El juguete y su didáctica», La Escuela en Acción, I0.4I3 (1980).

Jovellanos, G. M.: Memoria sobre las diversiones públicas, Madrid, Imprenta de Sancha, I8г2. «Juegos y deportes. El marro», Niños de Escuela Española (suplemento infantil de la revista Escuela Española), 59 (1948).

«Juegos en la Escuela», Boletín de la Inspección de la Enseñanza Primaria, 24 (1964).

«Juegos populares autóctonos y tradicionales», Conselleria de Educación e Ordenación Universitaria, Decreto 245/1992 de 30 de xullo, polo que se establece o currículo de Educación Primaria na Comunidade Autónoma de Galicia (DOG n. ${ }^{\circ}$ I58 venres I4 agosto 1992).

«Juegos de la Infancia». Segunda Parte: Madrid, Suc. de Hernando, s.a.

«Juegos tradicionales y populares», Generalitat de Catalunya, Departament d'Ensenyament, Decreto 95/1992, de 28 de abril, por el cual se establece la ordenación curricular de la educación primaria (DOGC 1593, de I3 de maig de 1992).

JunTa de Andalucía, Decreto/rg92 de por el que se establecen las enseñanzas correspondientes a la educación primaria en Andalucía (BOJA n. ${ }^{\circ} 56$ de 20 de junio de 1992).

Jurado, J. J.; Yague, V. y López, M.: Juegos de Madrid: los juegos populares y tradicionales como recurso didáctico, Madrid, Caja Madrid, 1998.

Kami, C. y Devries, R.: Juegos colectivos en la primera enseñanza. Implicaciones de la teoría de Piaget, Madrid, Visor, 1988.

«La enseñanza gimnástica», Diario de Las Palmas, 3 de abril de i894, p. 2.

«La enseñanza gimnástica en los institutos», Diario de Las Palmas, 3I de enero de i895, p. 2.

«La escuela y la educación física», Eco del Magisterio Canario, 30 de enero de 1919, pp. 3-4.

Lavega, P.: Del joc a l'esport. Estudi de les bitlles al Pla d'Urgel, tesis doctoral, Lleida, Universidad de Barcelona, I955.

Lavega, P.: Juegos y deportes populares-tradicionales, Barcelona, Inde, 2000.

Lavega, P.; Lagardera, F.; Molina, F.; Planas, A.; Costes, A. y De Ocariz, U. S.: «Los juegos y deportes tradicionales en Europa: Entre la tradición y la modernidad», Apunts Educación Física y Deportes, 85 (2006), pp. 68-8I. 
Lavega, P.; Moya, B.; Lagardera, F. y Freixa, C.: La litúrgia de les bitlles: Funcions $i$ sentit dun joc tradicional, Lleida, Pagés, 1997.

Lavega, P. y Olaso, S.: rooo juegos y deportes populares y tradicionales: La tradición jugada, Barcelona, Paidotribo, 1999.

«Las cuatro esquinas», La Aurora de la Vida, v. I, I6 (I86I), pp. $255-256$.

«Las cuatro esquinas», El amigo de la Infancia, v. 7, 74 (I880).

Lícito recreo Casero, ó colección de cincuenta juegos conocidos comúnmente con el nombre de juegos de Prendas: entretenimiento para pasar divertidas las largas noches de invierno con diferentes sentencias adecuadas para aumentar la diversión por un aficionado, Madrid, Imp. Ramón Ruiz, I792,

LinAzA, J. L.: Jugar y aprender (Documentos para la reforma, 7), Madrid, Alambra-Dogman, 1992.

Linaza, J. L.; Simón, C.; Sandoval, M. y García, M. G.: «Herramientas didácticas para la educación intercultural: el juego y las canciones», Aula Abierta, 80 (2005), pp. 27-42.

Llano, A.: «Rimas infantiles, escondite, cantares de corro, comba y otros juegos», en Esfozaya de cantares asturianos, Oviedo, Bibl. Popular asturiana, 1977.

Llorca García, A.: «Lo que deben ser los juegos de los niños», La escuela Moderna, X, II2 (I900).

«Lo que deben ser los juegos de los niños», La Región Canaria, I2 de julio de I90o, p. 2.

Lombardo-Radice, G.: «El folklore y la educación de los niños en Italia», Revista de Pedagogía, 8I (1928), pp. 385-386.

López, F.: Colección de Juegos para niños de ambos sexos, Madrid, Imp. Antonio Pérez Dubrull, 1855 .

López, R. y Garfella, P.: El juego como recurso educativo. Guía antológica, Valencia, Universidad de Valencia, I997.

López Santos, A.: Juegos, fiestas y diversiones en la América española, Madrid, Mapfre, 1992.

López SERra, F.: «Los juegos en la Institución Libre de Enseñanza: de I876 a I898», Ensayos: Revista de la Facultad de Educación de Albacete, 13 (1998), pp. 249-264.

«Lugares para juegos infantiles y grupos escolares», Diario de Las Palmas, 3I de enero de I928, p. I.

Luzuriaga, L.: «El juego y el trabajo en la educación», Revista de Pedagogía, viII, 92 (1929).

Machado y Álvarez, A.: «Juegos infantiles españoles, Boletín de la Institución Libre de Enseñanza, t. VIII, I75 (I884).

Machado y Álvarez, A.: «Terminología del Folk-Lore, por Mr. E. Sidney Hartland», Boletín de la Institución Libre de Enseñanza, I89 (1884), pp. 38I-383.

Machado y Álvarez, A.: «Juegos infantiles españoles. Dedicado al insigne mitógrafo italiano Sr. D. José Pitrè», Boletín de la Institución Libre de Enseñanza, I75 (I884), pp. I49-I54

Machado y Álvarez, A.: «Terminología del Folk-Lore, observaciones de C. Stanislad Wake; Observaciones de Henry B. Wheatley y G. Laurence Gomme», Boletín de la Institución Libre de Enseñanza, 193 (I885), pp. 6I-62.

Machado y Álvarez, A.: «Ensayo de juegos y canciones infantiles de Antonio Gianandrea», Boletín de la Institución Libre de Enseñanza, 194 (1885), pp. 73-79.

Machado y Álvarez, A.: «Los juegos de los salvajes. Traducción de The Saturday Review, con notas de M», Boletín de la Institución Libre de Enseñanza, 206 y 207 (I885), pp. 270-272 y pp. 282-283.

Maíllo, A.: «La educación del sentido social. El papel de los juegos», Servicio, 343 (1950).

Mangas Navas, J. M. «Vías pecuarias», Cuaderno de la Trashumancia, n. o o, Madrid, ICona. Ministerio de Agricultura, Pesca y Alimentación, p. I75.

Marcos Arévalo, J.: «El folklore desde la antropología cultural», Revista de Estudios Extremeños, 43 (3) (1987), pp. 645-66o.

Marín, I.: Jocs populars. I tu a què jugues?, Barcelona, Rosa Sensat, 1994.

Marín, I.: Juegos populares: jugar y crecer juntos, Barcelona, MEc, I995. 
Marín, I. y Pardo, O.: El jugar de l'Amades. Cultura d'infants. Volume: Moixanes, cançons de sorteig i jocs, Tarragona, Edicions El Mèdol, 1996.

Martí Alpera, F.: «Lo que deben ser los juegos de los niños», La Escuela Moderna, x, ino (I900).

MARTí, J.: «La tradición evocada: folklore y folklorismo», Tradición oral, I (1999), pp. 8I-Io8.

Martín, A.: El mundo del juguete. Cómo elegir, La Coruña, Adarra, 1976.

Martín Cebrián, J. y Martín Cebrián, M.: Juegos infantiles, Valladolid, Castilla Ediciones, I955.

Martín Nicolás, J. C.: Juegos tradicionales y deportes autóctonos de Castilla y León, León, Universidad de León, 2002.

Martínez, A.: «Indicación de los juegos infantiles más comunes y consideraciones sobre su valor educativo, Buñol», ADPV, E.9.3.15/caja 2, p. 20.

Martínez, E.: El juego infantil. Análisis escolar, Zaragoza, Universidad de Zaragoza, 1993.

Maspons, F.: Jocs d'Infants, Barcelona, Barcino, 1933.

MaYOR, A.: Historia de la Educación Física Infantil en el siglo XIX, Alcalá de Henares, Universidad de Alcalá, 2002.

MedinA, A.: «Maternales y párvulos. Juegos y juguetes», Vida Escolar, 4 (1959).

Medina, A.: «Juegos educativos», Vida Escolar, I3 (1959).

Medina, A.: Pinto Maraña. Juegos populares infantiles, Madrid, Miñón, 1987.

Medina, M. del C.: Deportes y juegos populares en Toledo, Toledo, Mc Medina, 1987.

Medina, M. del C.: Los juegos populares e infantiles en Castilla-La Mancha, Toledo, Servicio de Publicaciones de la JCCM, 20oI.

Menéndez, E.: «Aproximaciones al estudio de un juego: la rayuela. (Análisis etnológico)», Cuadernos del Instituto Nacional de Antropología, Buenos Aires, n. ${ }^{\circ}$ (1963).

Miranda, J. G.: «El uso de juegos tradicionales en el proceso educativo y su desvirtuación en la praxis pedagógica», Contextos Educativos. Revista de Educación, I (2013), pp. 251-268.

Molina-Duque, F.; Planas, A.; Lagardero, F.; Lavega, P. y Sáez de Ocariz, U.: «Los juegos y deportes tradicionales en Europa: Entre la tradición y la modernidad», Apunts: Educación Física y Deportes, 85 (2006), pp. 68-8I.

Monforte, E.: Una reconstrucció del joc de pilota valenciana als pobles de la comarca de l'Horta Sud de València de I90o a 1975: una investigación des de la historia oral, tesis doctoral, Universitat de València, 2015.

Monjo, E. y Mayor, J.: Jocs populars valencians de la marina alta, Alicante, Instituto de Cultura Juan Gil-Albert, 1999.

Montalbán, R.: El corro de las niñas. Canciones populares infantiles, Madrid, Casa Romero, I894.

Montoto y Rautenstrauch, L.: Personajes, personas y personillas que corren por las tierras de ambas Castillas, Sevilla, librería de San José Francos, I9II.

Morales, A.: A la una, la rabia, la mula: Juegos de la infancia en Usagre, Badajoz, Diputación de Badajoz, 2002.

Moreno, C.; Mata, D. y Gómez, J.: Aspectos recreativos de los juegos y deportes tradicionales en España, Madrid, Gymnos, 1993.

Moreno Palos, C.: Juegos y Deportes tradicionales en España, Madrid, Gymnos, 1992.

Morera Castro, M.: "Generación tras generación, se recobran los juegos tradicionales», Mbsalud, 5 (I) (2008).

Naharro, V.: Descripción de los juegos de infancia, los mas propios a desenvolver sus facultades físicas, morales, y para servir de abecedario gimnástico, Madrid, Imp. que fue de Fuentenebro, I818.

NARgANES, J. C.: Juego y desarrollo curricular en educación física: orientaciones para la reforma en enseñanza primaria, Sevilla, Wanceulen, 1993.

Navarro, V.: El afán de jugar, Barcelona, Inde, 2002. 
Navarro, V.: «40 años de propuestas de juegos motores. Análisis historiográfico de la estructura interna de las propuestas tradicionales y modernas de libros-manuales de juegos motores dirigidos a la Educación Física en Primaria», en XXII Congreso Nacional de Educación Física, A Coruña, 22-24 de septiembre de 2004.

Nelson, E.: «Función educativa y social de la plaza de juegos», Boletín de la Institución Libre de Enseñanza», LVII, 873 (1933).

Niño y VIÑAs, L.: Educación física. Ejercicio corporal pedagógico para escuelas normales y de niños), Salamanca, Imprenta Católica Salmanticense y encuadernación, I9I5.

«Notas pedagógicas. El juego educativo», El Tiempo, n. ${ }^{\circ}$ 1337, 6 de septiembre de 1907, p. I.

«Notas y observaciones. Trabajos manuales», El Tiempo, n. ${ }^{\circ}$ 1394, I2 de noviembre de 1907, p. I

Número monográfico «Especial El juego», Educación Infantil, I0.586 (200I).

Öfele, M. R.: «Los juegos tradicionales en la escuela. Primera y segunda parte», Revista Educación Inicial, Buenos Aires. año I3, II9-I20 (1988).

ÖFELE, M. R.: «Los juegos tradicionales y sus proyecciones pedagógicas», Lecturas: Educación Física y Deportes, 4 (13) (1999), pp. I-I5.

Olle, F.: «El juego y los juguetes», Revista de Educación, 19I (1967).

Orden de i6 de marzo de I970 por la que se aprueba el Estatuto del jugnete de interés pedagógico (BOE, 7 de abril de 1970).

Omeñaca Cilla, R. y Ruiz Omeñaca, J. V.: Juegos cooperativos y Educación Física, Barcelona, Paidotribo, 1999 .

OrLICK, T.: Juegos y deportes cooperativos, Madrid, Editorial Popular, 1996.

ORTEGA, R.: Jugar y aprender: una estrategia de intervención educativa, Sevilla, Díada editoras, 1990.

Ortiz García, C.: «La antropología pedagógica en España durante el primer tercio del siglo XX», Revista de Dialectología y Tradiciones Populares, 58 (2) (2003), pp. 7I-92.

Osés, J.: Juegos de campo, propios para niños y jóvenes, Barcelona, Tip. Perelló Vergés, I9I4.

Osorio, F. A.: Juego del palo, Edirca, 1980.

Panero, J. A. y Bernardo, H. A.: Juegos tradicionales sayagueses, Bermillo de Sayago, Zamora, Aderisa, 2007.

Parlebas, P.: Contribution á un lexique commenté en science de l'action motrice, Paris, INSEP, I98I.

Parlebas, P.: Elementos de Sociología del deporte, Málaga, Unisport, 1988.

Parlebas, P.: Juegos, deporte y sociedad. Léxico de praxiologia motriz, Barcelona, Paidotribo, 200I.

Parlebas, P.: «Lectura profunda de los cuadros de Brueguel el Viejo y Stella desde la praxiología motriz», en Actas del VIII Seminario Internacional de Praxiologia Motriz, Universidad de Murcia, 2003, pp. 9-36.

Parlebas, P.: «El destino de los juegos: herencia y filiación», en Lagardera, F. y Lavega, P. (coords.): La ciencia de la acción motriz, Lleida, Universitat de Lleida, 2004, pp. 59-76.

Pastor, J. L.: Educación física y libros de texto en la España contemporánea (I883-1978), Alcalá, Universidad de Alcalá, 2004.

Pavía, V. A.: «Investigación y juego, reflexiones desde una práctica», Revista Digital EFdeportes, Buenos Aires, año 5, n. ${ }^{\circ}$ I8 (2000).

PAYÀ, A.: «El joc a l'escola i els Mestres valencians de principis de segle XX», en vv. AA.: La renovació pedagógica, Girona, CCG Edicions, 2003.

PAYÀ, A.: «Joc corporal, esport i educación física a l'ideari pedagògica de la Institución Libre de Enseñanza», Educació i Història. Revista d'Història de l'Educació, 7 (2004), pp. II7-I33.

PAYÀ, A.: La actividad lúdica en la historia de la educación española contemporánea, Valencia, Servei de Publicacions de la Universitat de València, 2007.

PAYÀ, A.: «Consideraciones pedagógicas sobre los valores y posibilidades educativas del juego en la España contemporánea (1876-1936)», Historia de la Educación. Revista Interuniversitaria, 26 (2007), pp. 299-325. 
PAYÀ, A.: «El juego como recurso de educación moral y cívico-social», Revista de Ciencias de la Educación, 209 (2007), pp. 75-100.

PayÀ, A.: Aprender jugando. Una mirada bistórico-educativa, Valencia, Universitat de València, 2008.

PAYÀ, A.: «Joc i cultura del poble valencià: dos textos dels segles XVII i XVIII», Temps d'Educació, 34 (2008), pp. 205-220.

PAYÀ, A.: «Jugar a la guerra i a la pau. Breu història dels discursos ludicopedagògics en els segles XIX i XX», Aloma. Revista de psicología, ciències de l'educació $i$ de l'esport, 25 (2009), pp. II9-I36.

PAYÀ, A.: «O xoguete educativo español no século xx», Revista Galega de Educación, 5I (20II), pp. 44-47.

PaYÀ, A.: «Aprender deleitando. El juego infantil en la pedagogía española del siglo XX», Bordón, vol. I, 65 (2013), pp. 37-46.

PAYÀ, A.: «Juego, juguete y educación en la pedagogía española contemporánea», Espacios en Blanco, 24 (2014), pp. I07-I26.

PayÀ, A.: «El juego y el juguete en la historia de la escuela», en Mayordomo, A. y BArberà, Ó. (eds.): Escoles i mestres: dos siglos de historia y memoria en Valencia, Valencia, PUv, 2017, pp. I36-I52.

Payà, A. y Oliver, D.: «La infancia tiene cada vez menos tiempo para jugar», El País, 27 de julio de 20I8, disponible https://elpais.com/elpais/20I8/07/II/mamas_papas/I531309495_2I2620. html.

Pelegrín, A.: Cada cual atienda su juego, Madrid, Editorial Cincel, 1984.

Pelegrín, A.: Juegos y poesía popular en la literatura infantil y juvenil (I750-1987), tesis doctoral, Madrid, Universidad Complutense de Madrid, Facultad de Filología, i992.

Pelegrín, A.: La flor de la maravilla: Juegos, recreos, retahilas, Madrid, Fundación Germán Sánchez Ruipérez, 1996.

Pelegrín, A.: Repertorio de antiguos juegos infantiles: Tradición y literatura Hispánica, Madrid, Consejo Superior de Investigaciones Científicas, 1998.

Pérez, A. J.: «Juego y tradición folklórica en una novela de Mario Vargas Llosa», Olivar, i8 (2OI2), pp. I-I9.

PÉrez, G.: «La pedagogía del juguete», Escuela Española, 2.845 (1986).

Pérez Verdes y Tabernero Balsa: Xogos Populares en Galicia, La Coruña, Xunta de Galicia, 1986. Pérez Vidal, J.: Folklore infantil canario, Madrid, Cabildo Insular de Gran Canaria, 1986.

Pino Abad, M.: El delito de juegos probibidos: análisis histórico-jurídico, Madrid, Dykinson, $201 \mathrm{I}$.

Plath, O.: Origen y folclor de los juegos en Chile, Santiago de Chile, Grijalbo, 1988.

Powell, B.: Juegos de explorador, Madrid, Tip. José Yagües, I9I4.

«Práctica de los juegos populares y tradicionales» País Vasco, Decreto 236/Ig92 de iI de agosto, por el que se establece el currículum de la Educación Primaria para la Comunidad Autónoma del País Vasco (BOPV de jueves 27 de agosto de 1992).

«Por el juego se inicia el niño en el trabajo», Eco del Magisterio Canario, 7 de septiembre de I927, pp. 3-5.

«Por la Jefatura del Servicio Nacional de Primera Enseñanza ha sido dirigida la siguiente circular a la Inspección de Primera Enseñanza y maestros nacionales, municipales y privados de la España Nacional», Gaceta de Tenerife, n. ${ }^{\circ}$ 9067, 18 de marzo de 1938, p. 3.

Pou, A.: Jocs populars, Palma de Mallorca, Editorial Moll, i98o.

Prellezo, J. M.: Diario del P. Manón I895-1905, Madrid, Biblioteca de Autores Cristianos, 1973.

Pulet, R.: Juegos de animación en educación infantil y primaria, Málaga, Aljibe, 1995.

QueYRat, F.: Los juegos de los niños. Estudio acerca de la imaginación creadora en el niño, Madrid, Ed. Daniel Jorro, I926.

RabAtÉ, C.: «Juegos y educación en algunas revistas madrileñas de mediados del siglo XIX», Historia de la Educación, I2-I3 (1993-1994). 
Ramírez, L. F.; Luchena, S.; Del Campo, F. y Delgado, J. R.: Juegos tradicionales de Puertollano y su comarca, Puertollano, Editorial Puertollano, 1998.

Ramos, L.: "Gira, trompo, gira...», Cuadernos de Pedagogía, 1996 (243).

Ramos, R.: «El baile del Matachín», Studia Aurea, Navarra, vol. II, 1996.

Recas, L. D.: Deportes tradicionales de fuerza en España, Editorial Visión Libros, 2014.

Rementería, M.: Manual completo de juegos de sociedad o tertúlia y de prendas, Madrid, Imp. de don Norberto Llorenci, I839.

Rementería, M.: Juegos de niños traducidos por los mejores manuales acabados de publicar en París, Madrid, Fonseca, I847.

Renson, R.: «El retorno de los juegos tradicionales», Rev. Educación Física, n. ${ }^{\circ} 8$ (199I).

REYES, G.: «Juegos pastoriles», en Tesoro de conceptos divinos compuesto en todo género de verso, Sevilla, Clemente Hidalgo, I6r3.

RodrigáñEz, S.: El juego de pelota al alcance de todos, Madrid, I983.

Rodríguez, A.: Juegos y canciones tradicionales, La Laguna, Centro de la Cultura Popular Canaria, 1987.

Rodríguez, F.: Pasatiempo folklórico. Varios juegos infantiles del siglo XVI, Madrid, Tipografía de Archivos, 1932.

Rodríguez, S.: «El juego y la escuela», Escuela Española, 399 (1949).

Rodríguez Cascos, O.: El juego de bolos en tierras leonesas, León, Ed. Nebrija, 1978.

Rodríguez López, S.: La Sección Femenina y la sociedad almeriense durante el Franquismo. De las mujeres del Movimiento al Movimiento Democrático de Mujeres. tesis doctoral, Universidad de Almería, 2004, pp. 596-597.

Romaní, A.: Xogos infantiles de Galicia, Santiago, Follas Novas, I979.

Romero, V. y Gómez, M.: Metodología del juego, Servicios socioculturales y a la comunidad CFGS Educación Infantil, Barcelona, Altamar, 2003.

Rosa Sánchez, J. J. y Río Mateos, E.: Glosario de juegos tradicionales, León, Universidad de León, 2000.

Rosa Sánchez, J. J. y Río Mateos, E.: Juegos tradicionales infantiles en León, León, Universidad de León, 20oI.

Rubio, R.: «Biblioteca de las tradiciones populares españolas», Boletín de la Institución Libre de Enseñanza, I82 (1884), pp. 271-272.

Rubio, R.: «Los juegos corporales en la educación», Boletín de la Institución Libre de Enseñanza, 394 (I893).

Rubio, R.: «El pasado y el porvenir de la educación física, según el Dr. Mosso», Boletín de la Institución Libre de Enseñanza, t. Xx, 436 (1896).

Rubio, R.: «Hay que enseñar a jugar», La Escuela Moderna, iı8 (I90I).

Ruiz, M. J.; Fraile, J. M. y Weich-Shahak, S.: Al vaivén del columpio: Fiesta, coplas y ceremonial, Cádiz, Universidad de Cádiz, 2008.

Ruiz, P.: «Indicación de los juegos infantiles más comunes y consideraciones sobre su valor educativo", Puebla de San Miguel, ADPV, E.9.3.15/caja 2, 1908.

Rusel, A.: El juego de los niños. Fundamentos de una teoría psicológica, Barcelona, Herder, 1970.

SAbater, A.: «Juegos infantiles», Boletín del Liceo Escolar, Lleida, II4-II5 (I9I8), pp. 2-5.

SAEgesser, F.: Los juegos de simulación en la escuela. Manual para la construcción y utilización de juegos y ejercicios de simulación en la escuela, Madrid, Visor, I99I.

Sáez, P.: «Los juegos de los escolares», Escuela Española, 2.518 (20I4).

SAinz Pardo, M.: «El juguete improvisado», Didascalia, 47 (1974).

SAn Martín, A.: «Los juegos corporales», La Escuela Moderna, x, Io8 (1900).

SÁnchez, R.: «Educación física», La Escuela en Acción, 7.504-7.505 (1947).

SÁnchez, R.: «Juegos escolares», La Escuela en Acción, 7.517-7.518 (1947).

SÁnchez, R.: «Juegos escolares», La Escuela en Acción, 7.762-7.763 (1950).

SÁnchez, R.: «Los juegos escolares», El Magisterio Español, 7.754-7.775 (1950). 
SÁnchez, R. F.: «Una clasificación de los juegos tradicionales en la Alcarria Baja», Cuadernos de Etnología de Guadalajara, 47 (2015), pp. 337-354.

SÁnchez, S.: La lucha canaria y otras luchas del mundo, Tenerife, 1985.

SÁnchez Arbós, M.: «Los problemas de la Escuela. Ix. El juego», Boletín de la Institución Libre de Enseñanza, 888 (1934), p. 75.

SAnchís SAnchís, J.: "Los valores del deporte en el año europeo de la educación a través del deporte», en Giménez Fuentes-Guerra, F. J.; Sáenz-López Buñuel, P. y Díaz Trillo, M. (eds.): Educar a través del deporte, Huelva, Servicio de publicaciones Universidad de Huelva, 2005.

Sans Mercadal, J.: Els meus jocs: La creació de jocs a partir del folklore i la cultura de les illes balears, Palma, Universitat de les Illes Balears, 2005.

Santamaría, F. J.: El juego de bolos en las sierras de Cazorla y Segura, un juego tradicional andaluz, Málaga, Unisport, 1987.

Santiago, A.: Lolita. Cantares y juegos de las niñas, Madrid, Hijos de Tello, ı9ıo.

SANTOS: Juegos de los niños en las escuelas y colegios, Madrid, Ed. Saturnino Calleja, I882.

SANZ, I.: Juegos populares en Castilla León, Valladolid, Castilla, 1983.

Sarabia, D. y Simal, M.: Juegos tradicionales de Cantabria, Santander, Fundación Marcelino Botín, 2004.

Schmitt, A.: «Prólogo», en Guillemard, G.; Marchal, J. C.; Parent, M.; Parlebas, P. y Schmitt, A.:Las cuatro esquinas de los juegos, Lleida, Agonos, 1988.

Secadas, F.: «El juego en el ciclo inicial», Bordón, 237 (198I), pp. I43-I64.

Seirul.lo Vargas, F.: «Valores educativos del deporte», en Blázquez Sánchez, D. (dir.): La iniciación deportiva y el deporte escolar, Barcelona, Inde, 1998, pp. 62-67.

Sela, A.: «La educación física de la mujer», Boletín de la Institución Libre de Enseñanza, t. XII, 285 (I888).

Silvestre, N. y Martínez, I.: "Tiempo de jugar. El juego en el parvulario», Cuadernos de Pedagogía, I (1975).

SLuYs, A.: «Los programas de educación física en la escuela belga», Boletín de la Institución Libre de Enseñanza, 6Io (I9II).

Sluys, A. y Joncheere, T.: «Los juegos en la educación», Boletín de la Institución Libre de Enseñanza, 645 (1913).

SMAll, M.: El niño actor y el juego libre de expresión, Buenos Aires, Kapelusz, 1962.

SuÁrez Figueroa, C.: Plaza universal de todas ciencias y artes, Madrid, Luis Sánchez, I6I5.

SuARi Rodríguez, C.: «Sobre la repercusión de la deportificación del juego Tradicional», Lecturas: Educación Física y Deportes, 93 (2006).

TABernero, B.: Juegos del pasado en el presente y otras experiencias jugadas con personas mayores, Salamanca, Universidad de Salamanca-Ayuntamiento de Salamanca, 20 I2.

TAVERner, J. A.: «Los juegos en la enseñanza», Escuela Española, 5 I (1942).

TAYlor, A. R.: «Los instintos y los juegos de los niños», La Escuela Moderna, Io8 (I900).

Tissié, Ph.: La fatiga y el adiestramiento físico, Madrid, Librería de Fernando Fé, I899.

Torrebadella, X.: Contribución a la historia de la educación física en España. Estudio biobibliográfico en torno a la educación física y el deporte (I800-1939), tesis doctoral, Lleida, Universitat de Lleida, 2009.

Torrebadella, X.: «Vicente Naharro y los juegos corporales en la Educación Física española de la primera mitad del siglo XIX», Ágora para la educación física y el deporte, vol. I3, 2 (20II), pp. I65-I8I.

Torrebadella, X.: «La educación física comparada en España (I806-1936)», Historia Social y de la Educación, 3 (I) (20I4), pp. 25-53

Torroja, R.: «Los juegos en la escuela», Revista de Pedagogía, 24 (1923).

Tourtet, L.: Jugar, soñar, crear, Salamanca, Sociedad de educación Atenas, 1973. 
Trigo, E.: Aplicación del juego tradicional en el currículum de educación física (Volumen I), Barcelona, Paidotribo, 1994.

Trigo AzA, E.: «El juego tradicional en el currículum de Educación Física», Revista Aula de Innovación Educativa, Barcelona, n..$^{\circ} 44$ (1995), pp. 20-24.

Trigueros, C.: Nuevos significados del juego tradicional en el desarrollo curricular de la educación física en centros de Educación Primaria en Granada, tesis doctoral, Granada, Universidad de Granada, 2000.

Trillo, M.: «Para las Escuelas Maternales y de Párvulos. Juegos educativos», La Escuela en Acción (suplemento de El Magisterio Español), 8.45I-8.452 (1956).

Tillo, M.: «Juegos educativos», La Escuela en Acción (suplemento de El Magisterio Español), $8.4519-8.460$ (1956).

Troncoso, J.: «Juegos y deportes. La educación física al servicios de la salud», El Magisterio Español, 6.670 (1939).

Tusquets, J.: «Juegos nacionales y pedagogía del desarrollo», Revista de Educación, I85 (1966). Un Papá: Los Juegos de la Infancia, París, Garnier Hermanos, I897.

UNESCo: El niño y el juego. Planteamientos teóricos y aplicaciones pedagógica, París, UnesCo, 1978.

VAREY, J. E.: «Representaciones de títeres en teatros públicos y palaciegos: I2II-I760», Revista de Filología Española, Madrid, vol. 28 (1954).

Veiga, P.: O libro dos xogos populares galegos: Catálogo descriptivo e educativo, Santiago de Compostela, Gotelo Blanco, 2002.

Velasco Maíllo, H.: «El folklore y sus paradojas», ReIS: Revista Española de Investigaciones Sociológicas, 49 (1990), pp. I23-I44.

Veleda, M. J.: Reglamentos de los deportes autóctonos de Castilla y León, Valladolid, Caja España, 1998.

VeledA, M. J.: Juegos infantiles tradicionales, Valladolid, Diputación Provincial de Valladolid, I999.

VIAL, J.: Juegos y educación. Las ludotecas, Madrid, Akal, 1988.

Vilanou, C. y Bantulà, J.: «Sobre la eutrapelia o la virtud del juego: moralidad, historia y educación», Bordón. Revista de Pedagogía, vol. 65, I (2013), pp. 47-58.

VIllán, J.: Tole, catole, cuneta: Juegos de la infancia perdida, Madrid, Akal, 1999.

Vives, J. L.: Diálogos de Juan Luis Vives, traducción y correcciones por Cristoval Coret, I759.

Vizuete, M. y Gutiérrez, J.: Juegos populares extremeños, Mérida, Editora regional de Extremadura, 1986.

vv. AA.: Xogos populares en Galicia, La Coruña, Xunta de Galicia, 1986.

VV. AA.: V Jornadas desafíos del juguete en el s. XXI. Juegos y juguetes para los contenidos escolares, Valencia, Fundación crecer jugando, 2003.

vv. AA.: Propuestas metodológicas para la enseñanza de los juegos y deportes autóctonos y tradicionales canarios, Consejería de Educación, Cultura y Deportes del Gobierno de Canarias, Las Palmas de Gran Canaria, 1997.

vv. AA.: Los juegos en la Educación Física de los 6 a los 12 años, Barcelona, Editorial Inde, 1998. $\mathrm{X}$ : «Juegos corporales», Boletín de la Institución Libre de Enseñanza, 206 (1886), pp. 43-46.

YÁñEz, A.: Flor de juegos antiguos, México, Grijalvo, 1977.

Zamora, M. D.: «El niño y el juego en la edad preescolar», Didascalia, 47 (1974). 
